\title{
Distinguishing between toxic and non-toxic pyrrolizidine alkaloids and quantification by liquid chromatography-mass spectrometry
}

\author{
Gertruida M. Rösemann, Christo J. Botha, Jacobus N. Eloff ${ }^{\star}$ \\ Phytomedicine Programme, Department of Paraclinical Sciences, Faculty of Veterinary Science, University of Pretoria, \\ Private Bag X 04, Onderstepoort, 0110, South Africa.
}

${ }^{*}$ Corresponding author. Tel +27 12529 8244; fax +27 125298304

E-mail address: kobus.eloff@up.ac.za (J.N. Eloff)

\begin{abstract}
Pyrrolizidine alkaloids (PAs) are important plant toxins causing poisoning in livestock, leading to significant financial and production losses each year. It may also enter the human food chain as contaminants of grains, via animal products such as milk, eggs and honey or herbal remedies. Not all PAs are toxic and it is important to be able to distinguish between toxic and non-toxic PAs. We developed a sensitive and selective analytical method to determine toxic 1,2-unsaturated PAs concentrations in plant extracts by liquid chromatography-mass spectrometry (LC-MS) with electrospray ionization and precursor ion experiments. Multi-reaction-mode experiments were used to quantify the concentrations of the different unsaturated PAs and results were expressed as $\mu \mathrm{g} / \mathrm{g}$ retrorsine equivalents. The results obtained on Crotalaria sphaerocarpa contaminating maize crops have important implications.
\end{abstract}

Keywords: Crotalaria; food contaminant; LC-MS detection; method development; 1,2-unsaturated pyrrolizidine alkaloids; Senecio toxicity 


\section{Introduction}

Generally, pyrrolizidine alkaloids (PAs) are esters of hydroxylated methyl pyrrolizidines, consisting of a necine base- and necic acid moiety. The necine base can either be 1,2-unsaturated or saturated. The unsaturated necine bases are further classified as two types, viz. retronecineand otonecine-type alkaloids (Ober and Hartmann, 1999). Pyrrolizidine alkaloid bases can also exist as $\mathrm{N}$-oxides, which are often present with the basic alkaloids in plants. Together with the $N$ oxides more than 640 pyrrolizidine alkaloid structures are possible, of which at least 350 types have already been found in nature and their structures elucidated (Mattocks, 1986).

Not all PAs lead to the synthesis of the toxic metabolite. Only the esters of 1,2-unsaturated retronecine- and otonecine type PAs, which are bioactivated by hepatic cytochrome PF mixed function oxidases to toxic dehydropyrrolizidines (DHP) are toxic (Prakash et al., 1999). These bifunctional DHP molecules can react with a variety of nucleophilic intracellular macromolecules, resulting in liver and other tissue damage associated with different types of PA poisonings (Fu et al., 2002). According to Wiedenfeld (2011) "Pyrrolizidine alkaloids (PAs) possessing a 1,2-double bond in their base moiety (necine) are hepatotoxic, carcinogenic, genotoxic, teratogenic and sometimes pneumotoxic".

Various analytical techniques have been used to separate, identify and quantify PAs in plants. However, for most of these procedures authenticated reference materials are needed, of which only a few are currently commercially available. Analytical methods that can selectively detect the $1,2-$ unsaturated necine bases of toxic PAs in complex mixtures are essential to estimate potential toxicity and to confirm a diagnosis of suspected poisonings (International Programme on Chemical Safety).

Lin et al. (1998) developed a LC-MS-MS method for the determination of known PAs. Spectra were obtained with in-source collision as well as with collision induced dissociation (CID) in the collision cell. All PAs analyzed by electrospray ionization (ESI) in the positive mode had an abundance of the $[\mathrm{M}+\mathrm{H}]^{+}$pseudo-molecular ion. CID spectra of retronecine-type 1,2-unsaturated PAs produced characteristic fragments at $\mathrm{m} / \mathrm{z} 120$ and 138. Other fragments characteristic of this 
type of PA were an ion at $\mathrm{m} / \mathrm{z} 94$ and a fragment corresponding to $[\mathrm{MH}-\mathrm{CO}]^{+}$. Triple quadrupole MS-MS detectors can be used in precursor scan mode, where the fragments produced after CID are used to determine the compound of origin. For diverse compounds like toxic PAs, where all the 1,2unsaturated structures yield such distinguished fragments, this would be the ideal screening method for the presence of 1,2-unsaturated toxic PAs in natural products or food. Our aim was to develop a quantitative screening method to search for unknown toxic PAs in various plants. In an attempt to evaluate the method four South African plant species were investigated:

1. Crotalaria sphaerocarpa seeds, associated with grain contamination (Eloff et al., 2003).

2. Crotalaria dura, commonly accepted as a poisonous plant in South Africa.

3. Crotalaria laburnifolia, not known to be toxic.

4. Senecio inaequidens, analyzed after suspected poisoning of cattle (Dimande et al., 2007).

\section{Materials and Methods}

\subsection{Chemicals}

Unsaturated retronecine-type PAs, i.e. retrorsine (CAS: 480-54-6) and monocrotaline (CAS: 315-22-0) were purchased from Sigma Aldrich (South Africa). Acetonitrile, ammonium acetate, hydrochloric acid, ethanol, zinc powder, ethyl acetate, ammonia solution $25 \%$, hexane and methanol were purchased from Merck (Darmstadt, Germany).

\subsection{Instrumentation}

A Waters Alliance 2796 HPLC gradient pump system (Microsep, South Africa) was used with a Phenomenex Luna C18 $5 \mu \mathrm{m} 250 \times 2.0 \mathrm{~mm}$ (Separations, South Africa) analytical column. Mobile phase A contained 90\% $25 \mathrm{mM}$ ammonium acetate buffer ( $\mathrm{pH} 3.84$ ), 2\% methanol and 8\% acetonitrile. Mobile phase B contained $80 \%$ acetonitrile, $10 \%$ methanol and $10 \% 25 \mathrm{mM}$ ammonium acetate buffer ( $\mathrm{pH} 3.84)$. Gradient elution was $0-5 \min 98 \%$ A; $5-15 \min 40 \%$ A (linear); $15-20 \mathrm{~min} 98 \% \mathrm{~A}$ (linear). The flow rate was $0.2 \mathrm{~mL} / \mathrm{min}$. Total runtime was 30 minutes 
with a 5 minute equilibration time at the end of the run. A Quattro Micro Triple Quad instrument (Micromass, Microsep, South Africa) mass spectrometer with electrospray interface in the positive mode was used with Argon as the collision gas for MS-MS experiments. The software used was MassLynx® version 4.0.

\subsection{Instrument optimization}

Solutions of pure reference materials - retrorsine (FW 351) and monocrotaline (FW 325) in methanol $(0.5 \mathrm{mg} / \mathrm{mL})$ - were infused to optimize the mass spectrometer settings in the MS (Fig. 1),
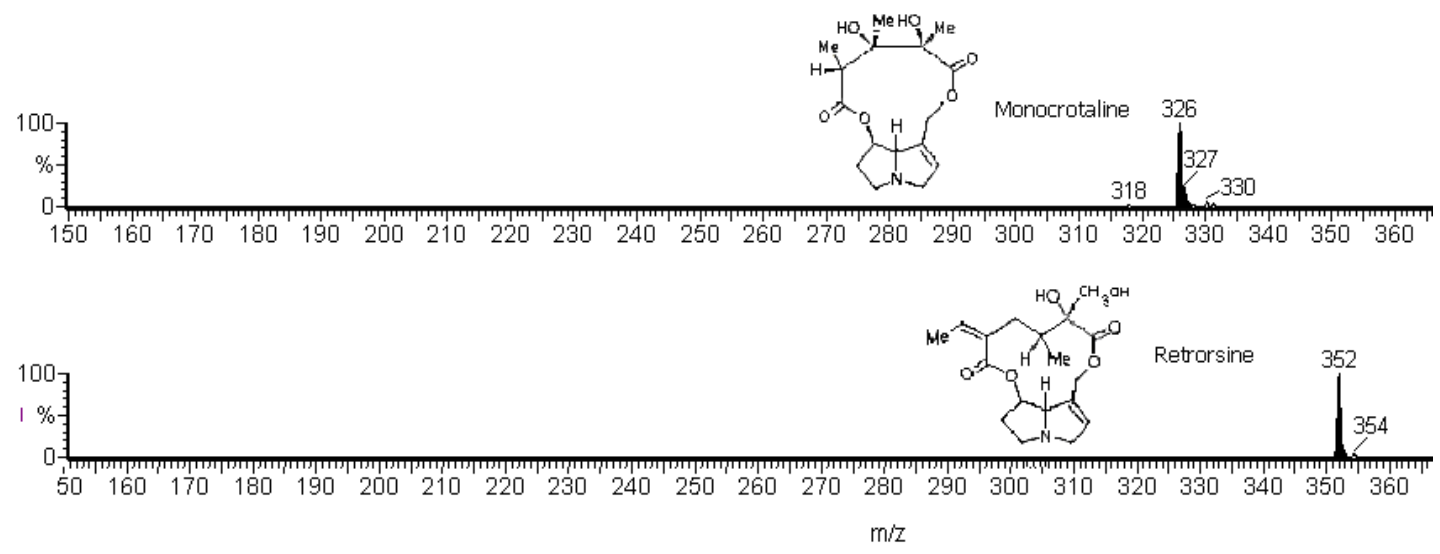

Figure 1. $\mathrm{ESI}^{+}$mass spectra of monocrotaline and retrorsine showing the $[\mathrm{M}+\mathrm{H}]^{+}$ions obtained with the infusion experiments.

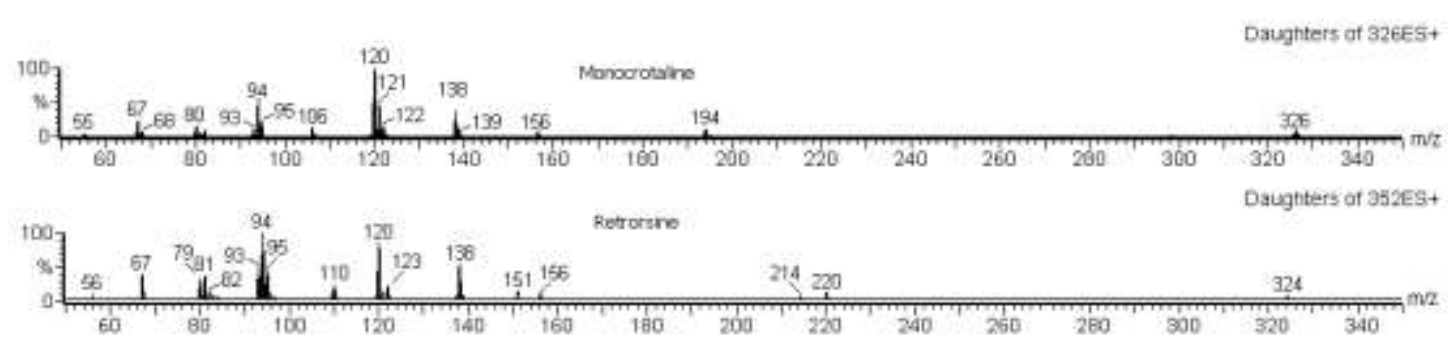

Figure 2. MS-MS spectra of pure monocrotaline and retrorsine with collision induced fragmentation in the collision cell (collision gas $=34$ ) showing the fragments characteristic of unsaturated PAs.

MS-MS (Fig. 2) and tandem LC-MS-MS modes. LC-MS-MS settings were optimized for the fragment m/z 120 and used for precursor scans (Fig. 3), product ion scans and multi-reaction mode (MRM) scans, performed on all the extracts. The MRM transition of the $[\mathrm{M}+\mathrm{H}]^{+}>m / z 120$ fragments were used for quantification. The LC-MS-MS settings were: Capillary $3.2 \mathrm{~V}$, Cone $20 \mathrm{~V}$, Extractor $3 \mathrm{~V}, \mathrm{RF}$ 
lens $0.3 \mathrm{~V}$, Source temperature $150^{\circ} \mathrm{C}$, Desolvation temperature $350^{\circ} \mathrm{C}$, Cone gas $60 \mathrm{~L} / \mathrm{h}$, Desolvation gas $300 \mathrm{~L} / \mathrm{h}$, Collision energy 34.

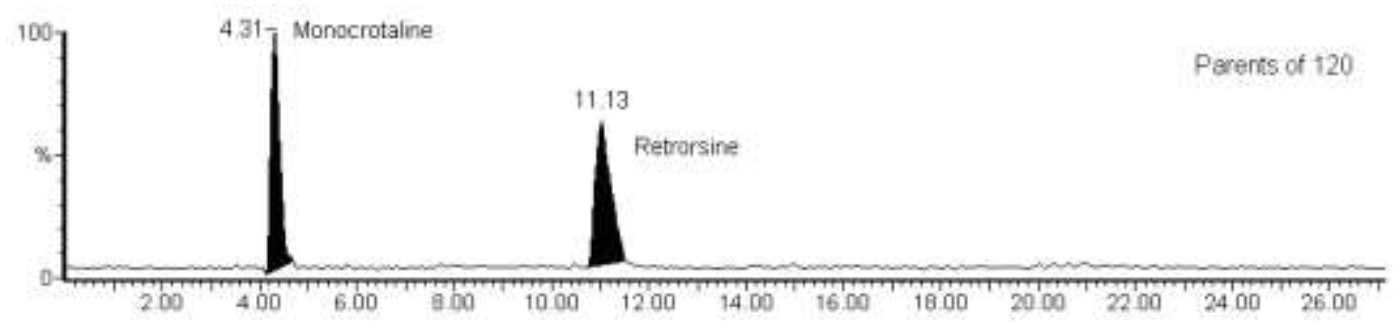

Figure 3. The LC-MS-MS total ion chromatogram (TIC) obtained with a precursor ion scan for the fragment $\mathrm{m} / \mathrm{z} 120$ of an extracted standard.

\subsection{Preparation of standards}

Dried, milled lucerne (Medicago sativa) was extracted with ethanol as a blank matrix and spiked with pure retrorsine and monocrotaline $(1 \mathrm{mg} / \mathrm{mL}$ methanol $)$ reference material. Serial dilutions with the blank matrix were used to prepare a standard curve consisting of 12 different concentrations between $0.01 \mu \mathrm{g} / \mathrm{mL}$ and $100 \mu \mathrm{g} / \mathrm{mL}$. Aliquots $(1 \mathrm{~mL})$ of the standard solutions were evaporated and extracted as described under section 2.6.

\subsection{Plant samples and rumen content}

Crotalaria sphaerocarpa seeds were collected from a maize farm $2 \mathrm{~km}$ north of Oberholzer in the Free State Province, Republic of South Africa. Crotalaria dura and Crotalaria laburnifolia plant material were collected in KwaZulu-Natal, South Africa by Prof. TW Naudé, Faculty of Veterinary Science, Onderstepoort. In October 2004 nine adult cows died near Frankfort in the Free State Province after ingesting plants suspected of containing PAs. The plants were identified by the National Herbarium, South African National Biodiversity Institute as Senecio inaequidens (DC) (Dimande et al., 2007). Necropsies were performed on cattle that have died and rumen content (500 g) was collected and submitted for analysis together with the collected plant material. 


\subsection{Sample handling}

$\mathrm{N}$-oxides are often present in plants together with the basic PAs. To enhance extractability, the polar $\mathrm{N}$-oxides were reduced to the basic alkaloids before extraction. The extract was then divided into two fractions and the $\mathrm{N}$-oxides in one of the fractions were reduced by addition of zinc. The total alkaloid content was determined in the reduced fraction, while the second fraction was used to determine the basic alkaloids content. The $\mathrm{N}$-oxide content is represented by the difference between the total and the basic alkaloid fractions.

The plant samples were well mixed and milled to obtain a sub-sample of 100 to $500 \mathrm{~g}$ plant material. A portion of the sub-sample was weighed $(1.0 \mathrm{~g})$, homogenized with $10 \mathrm{~mL} 90 \%$ ethanol and left on a mechanical shaker for about $4 \mathrm{~h}$. The solids were allowed to settle and the sample was centrifuged. The clear solution was divided into equal fractions $A$ and $B$ and evaporated at $40^{\circ} \mathrm{C}$ under reduced pressure. The standard and sample extracts were reconstituted in $2 \mathrm{~mL}$ dilute hydrochloric acid $(0.1 \mathrm{M})$. Chlorophyll and waxes were removed by extracting with $2 \times 5 \mathrm{~mL}$ hexane and the hexane layer discarded. The $N$-oxides in sample fraction $B$ were reduced by addition of about $0.5 \mathrm{~g}$ zinc powder and stirred overnight. Both sample fractions and standard solutions were made basic $(\mathrm{pH}>9)$ by addition of about $0.5 \mathrm{~mL} 25 \%$ ammonia solution. The alkaloids were extracted 3 times with $5 \mathrm{~mL}$ ethyl acetate. The ethyl acetate extracts were combined, evaporated and the alkaloids stored dry at $-20^{\circ} \mathrm{C}$ until analysis. Standards and samples were reconstituted in $1.0 \mathrm{ml}$ methanol before LC-MS analysis.

\subsection{Linearity, analyte recovery and limit of quantification (LOQ)}

Linearity was evaluated by extracting four aliquots $(n=4)$ of each standard concentration and analyzing them using MRM scans for the transition $\mathrm{m} / \mathrm{z} 352>120$ (retrorsine). The peak area concentration graphs were linear over the range 0.05 to $100 \mu \mathrm{g} / \mathrm{mL}\left(R^{2}=0.9979\right)$. Analyte recovery was evaluated by measuring the concentrations of monocrotaline in fortified preparations. Monocrotaline concentration $(0.05$ to $100 \mu \mathrm{g} / \mathrm{mL})$, was determined using MRM scans for the transition $m / z$ 326 $>120$. The recovery of monocrotaline was linear $\left(R^{2}=0.9861\right)$ from above the LOQ to 50 $\mu \mathrm{g} / \mathrm{mL}$ when calculated as retrorsine equivalents. The LOQ was defined as the lowest concentration 
of analyte that could be measured using MRM experiments for the transition $[\mathrm{M}+\mathrm{H}]^{+}>120$ at a signal/noise ratio of $10: 1$. The $L O Q$ for both retrorsine and monocrotaline was $0.05 \mu \mathrm{g} / \mathrm{mL}$ (or $0.5 \mathrm{ng}$ "on column") with the extracted standards.

\section{Results and Discussion}

For the initial detection of possible toxic PAs in a specific plant sample, two injections of the extract were needed. Precursor experiments were performed on the first injection to determine the pseudo-molecular masses $\left([\mathrm{M}+\mathrm{H}]^{+}\right)$of the compounds which produced the $\mathrm{m} / \mathrm{z} 120$ and 138

\section{C. sphaerocarpa}

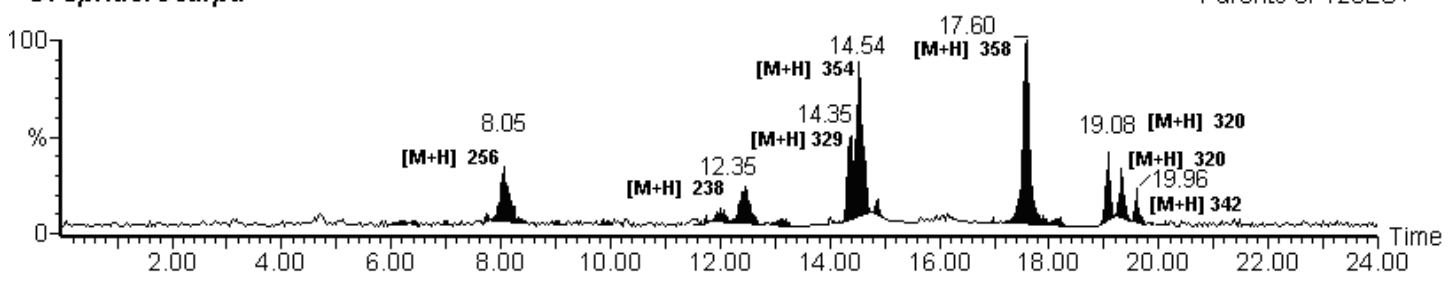

Figure 4. Precursor ion scan of Crotalaria sphaerocarpa seeds showing the pseudo-molecular masses of the different unsaturated PAs.

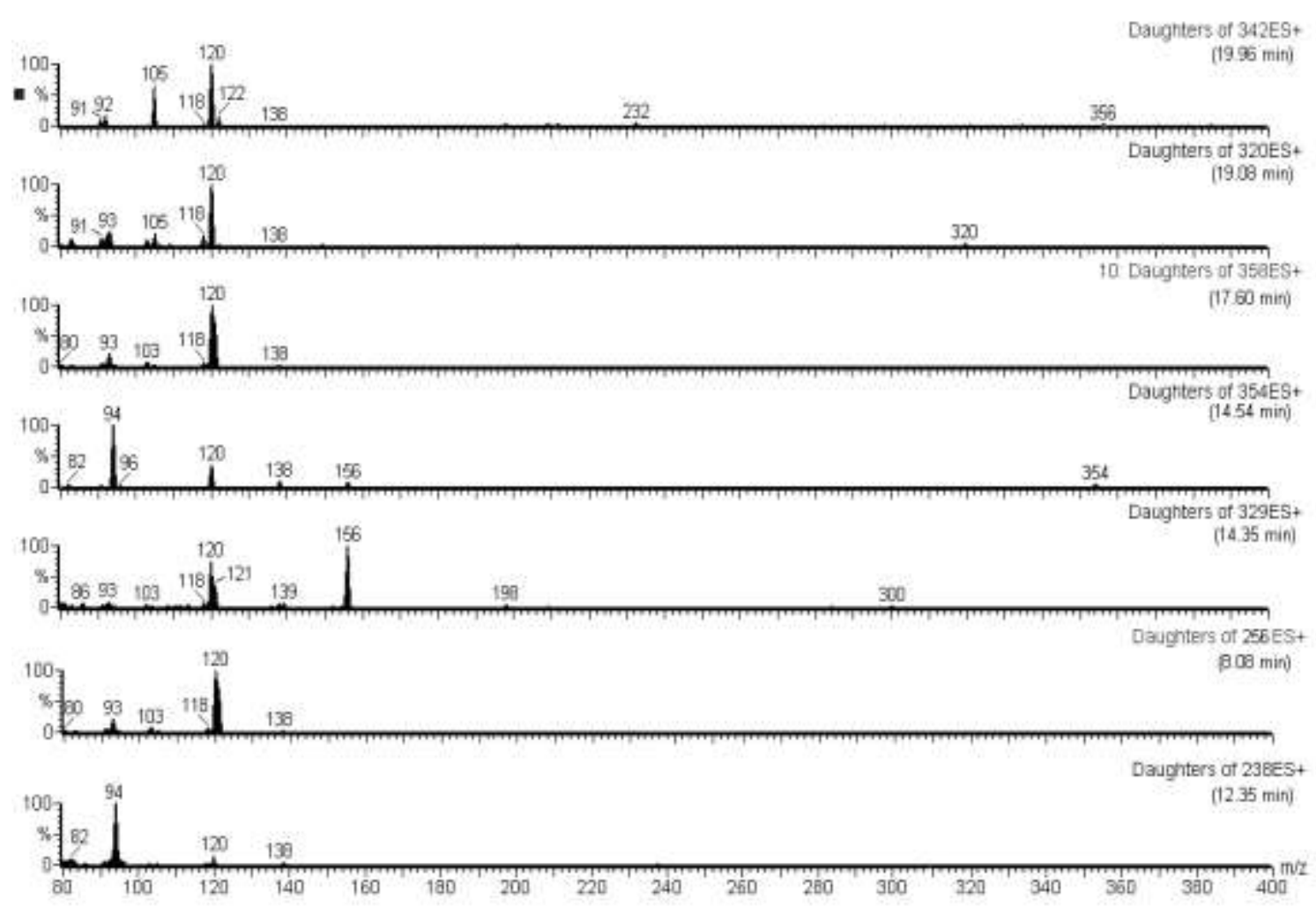

Figure 5. MS-MS spectra of the possible toxic PAs present in Crotalaria sphaerocarpa seeds. 
fragments (Fig. 4). In the second injection (Fig. 5) the detector was programmed to perform product ion scans over the mass range $\mathrm{m} / \mathrm{z} 50-400$, at the respective retention times of the $[\mathrm{M}+\mathrm{H}]^{+}$masses identified with the precursor scans. Compounds which revealed the characteristic mass fragments $\mathrm{m} / \mathrm{z} 120,138$ and 93-94 were accepted as originating from toxic unsaturated retronecine type PAs. MRM experiments were then performed on the transitions of the $[\mathrm{M}+\mathrm{H}]^{+}$mass of the compound and the fragment $\mathrm{m} / \mathrm{z} 120$ to quantify the different unsaturated compounds. The peak areas were used to calculate the concentration from the retrorsine calibration curve and results were expressed as $\mu \mathrm{g} / \mathrm{g}$ retrorsine equivalents.

Compounds which led to the expected characteristic fragments were quantified in subsequent MRM experiments. The concentration of the compounds were calculated against the retrorsine calibration curve and expressed as $\mu \mathrm{g} / \mathrm{g}$ retrorsine equivalents (Table 1 ).

Table 1. Concentration of toxic PAs in the samples investigated. Results were obtained using MRM experiments for the transitions of the precursor mass $>m / z 120$ and calculated against a retrorsine calibration curve. Results are reported as $\mu \mathrm{g} / \mathrm{g}$ retrorsine equivalents.

\begin{tabular}{|c|c|c|c|c|c|c|c|}
\hline \multicolumn{7}{|c|}{ Crotalaria sphaerocarpa seeds } & Total \\
\hline Retention time (min) & 8.0 & 12.6 & 14.4 & 14.5 & 17.6 & 19.5 & \\
\hline Pseudo-molecular mass & 256 & 328 & 329 & 354 & 358 & 320 & \\
\hline Concentration Basic PAs & 22.1 & 11.8 & 16.8 & 54.2 & 22.3 & 1.2 & 125.5 \\
\hline Concentration $\mathrm{N}$-oxides & 3.1 & $<1$ & 3.5 & 5.9 & 8.2 & 1.6 & 22.3 \\
\hline Total unsaturated PAs & 25.2 & 7.9 & 20.2 & 60.1 & 30.5 & 2.8 & 150.4 \\
\hline \multicolumn{8}{|c|}{ Crotalaria laburnifolia plant material } \\
\hline Retention time (min) & 4.2 & 14.1 & & & & & \\
\hline Pseudo-molecular mass & 282 & 338 & & & & & \\
\hline Concentration Basic PAs & 5.0 & $<1$ & & & & & 5.7 \\
\hline Concentration $\mathrm{N}$-oxides & 8.6 & 5 & & & & & 13.6 \\
\hline Total unsaturated PAs & 13.6 & 5.7 & & & & & 19.3 \\
\hline \multicolumn{8}{|c|}{ Crotalaria dura plant material } \\
\hline Retention time (min) & 3.2 & 4.3 & 13.0 & 14.1 & 18.0 & 20.8 & \\
\hline Pseudo-molecular mass & 282 & 282 & 324 & 340 & 350 & 393 & \\
\hline Concentration Basic PAs & 29.0 & 44.3 & 122.6 & 5.9 & 3.3 & 2.4 & 207.5 \\
\hline Concentration $\mathrm{N}$-oxides & 12.0 & 108.5 & 182.6 & 60.2 & 9.3 & 9.2 & 381.8 \\
\hline Total unsaturated PAs & 41.0 & 152.8 & 305.2 & 66.1 & 12.6 & 11.6 & 589.3 \\
\hline \multicolumn{8}{|c|}{ Senecio inaequidens plant material } \\
\hline Retention time (min) & 11.3 & 13.1 & & & & & \\
\hline Pseudo-molecular mass & 352 & 336 & & & & & \\
\hline Concentration Basic PAs & 234 & 19 & & & & & 253 \\
\hline Concentration $\mathrm{N}$-oxides & 11212 & 531 & & & & & 11743 \\
\hline Total unsaturated PAs & 11446 & 550 & & & & & 11996 \\
\hline
\end{tabular}


Eight different unsaturated PAs were detected in the extracted $C$. sphaerocarpa seed samples (Fig. 4).Two of the PAs (19.08 $\mathrm{min}$ and $19.96 \mathrm{~min}$ ) have the same $[\mathrm{M}+\mathrm{H}]^{+}$and spectrum, but is considered two separate compounds due to the difference in retention times. Product ion experiments were performed at the retention times where the precursors were found and the spectra (Fig. 5) were compared with the spectra of the pure PAs, obtained during the infusion experiments (Fig. 2). Seed samples from different regions of the country and stages of maturity were also analyzed with this method (data not shown here). The total unsaturated PA concentration varied considerably between seeds collected at different regions $(110 \mu \mathrm{g} / \mathrm{g} \pm 40)$, but the same pseudo-molecular ions were present in all the mature seed samples. The unsaturated PA concentrations of young (green) seeds were less than $4 \mu \mathrm{g} / \mathrm{g}$. These results have important implications for the number of $C$. sphaerocarpa seed pods that can be allowed in grain used for animal or human consumption (Eloff et al., 2003). The implications will be discussed in follow up papers on food safety.

\section{C. laburnifolia}

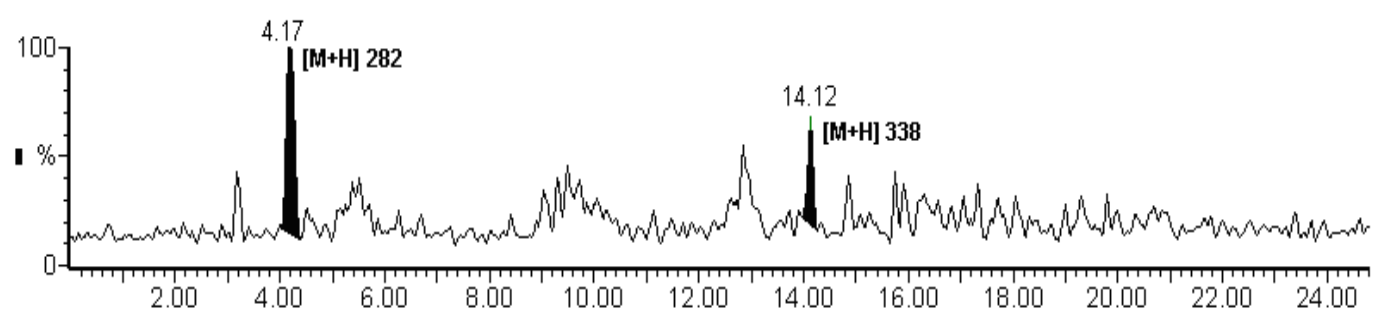

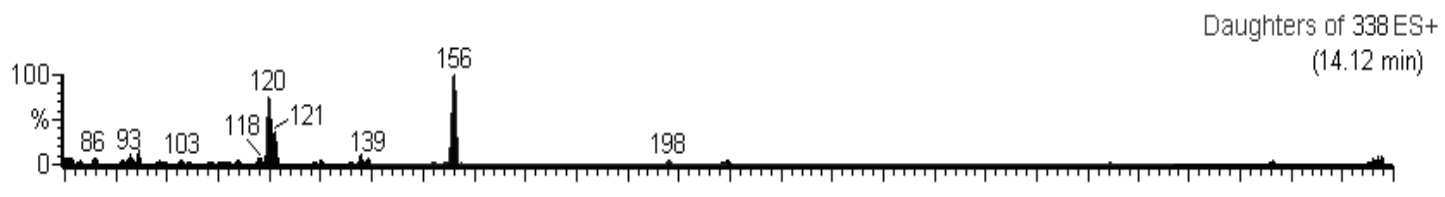

Daughters of $282 \mathrm{ES}+$

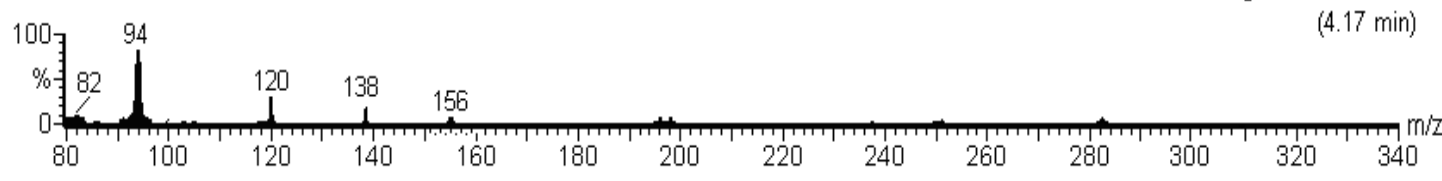

Figure 6. Precursor ion scan of Crotalaria laburnifolia plant extract with the MS-MS spectra of the unsaturated PAs.

Two unsaturated PAs were present in C. laburnifolia, with molecular masses 281 and 337 respectively (Fig. 6). The total unsaturated PA content in this plant was less than $20 \mu \mathrm{g} / \mathrm{g}$ and it is 
unlikely that this would be toxic to livestock, thus confirming the general belief that $C$. laburnifolia is non-toxic (Kellerman et al., 2005).

Crotalaria dura, on the other hand, is known to be pneumotoxic in horses, and have been shown in some instances to cause liver damage in horses, cattle and sheep (Kellerman et al., 2005). A total of six unsaturated PAs were found in this plant species with the LC-MS/MS method (Fig. 7) and the total unsaturated PA concentration was $590 \mu \mathrm{g} / \mathrm{g}$ and is most probably the cause of liver damage sometimes seen after ingestion of $C$. dura.
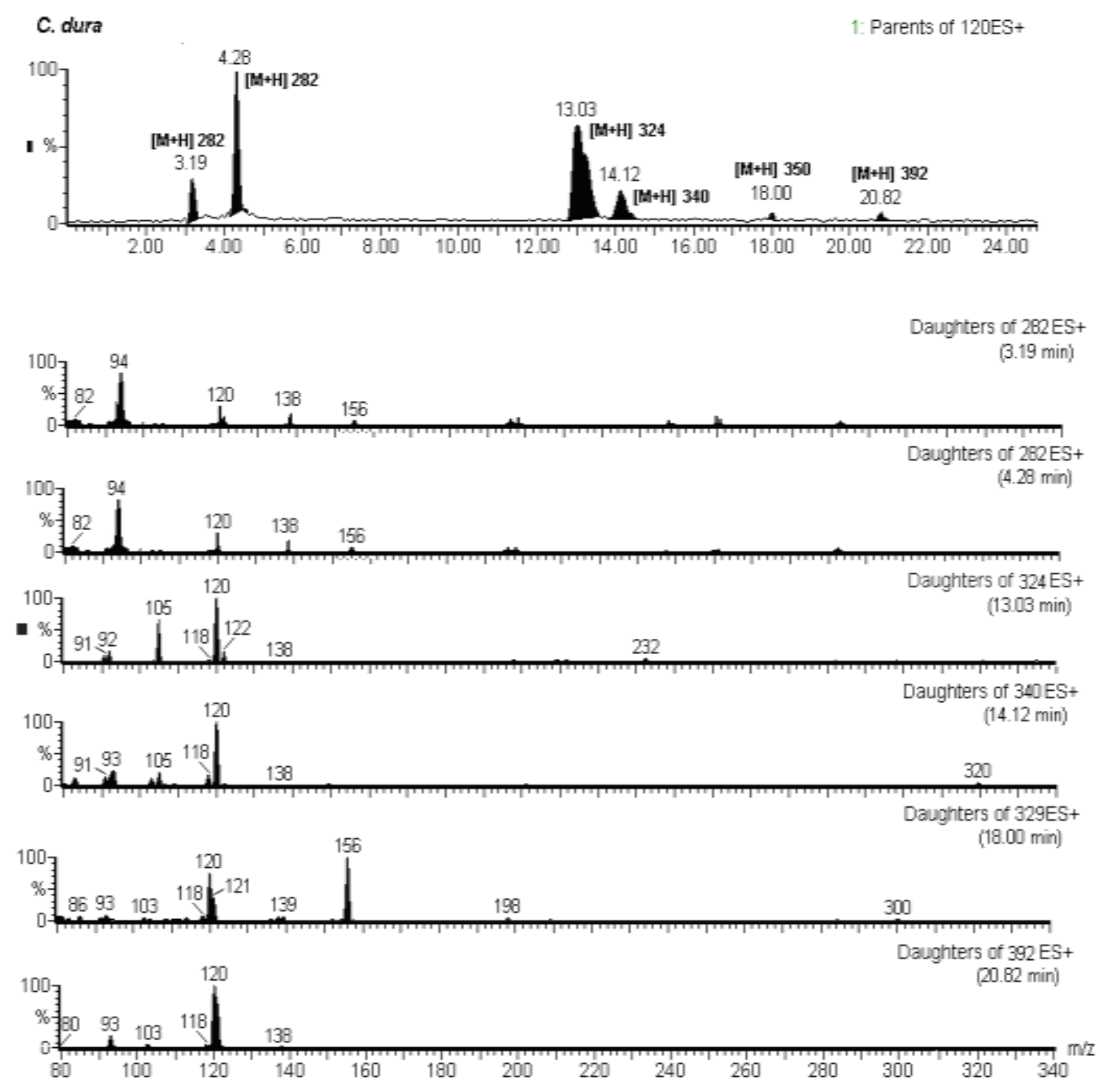

Figure 7. Precursor ion scan of $C$. dura plant extract with the MS-MS spectra of the compounds of interest.

Analysis of extracts prepared from the Senecio inaequidens plant material and rumen content collected following the cattle mortalities near Frankfort in the Free State Province, South Africa 


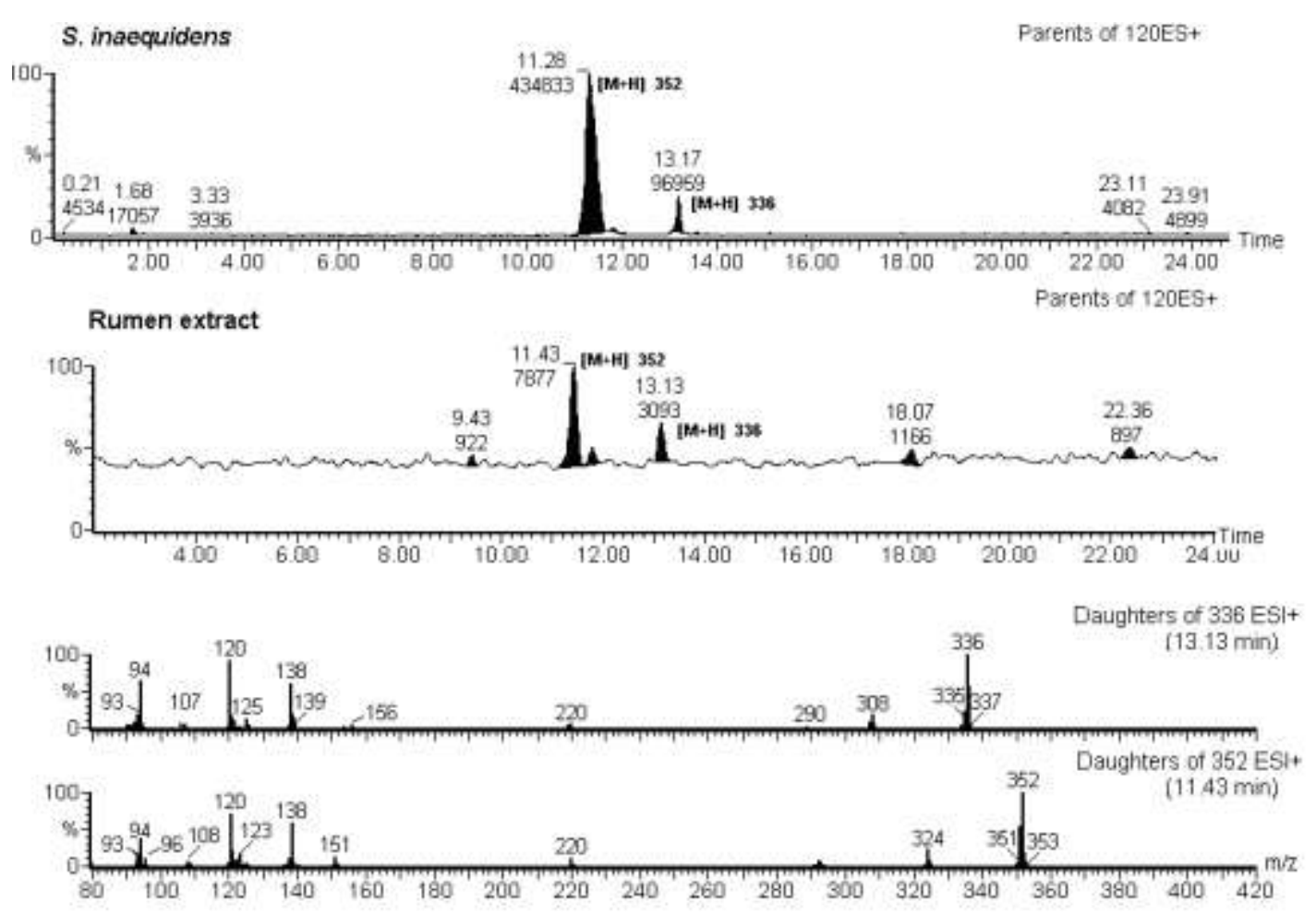

Figure 8. Precursor ion scan and MS-MS spectra of Senecio inaequidens plant extract and the rumen content collected postmortally with the MS-MS spectra of the unsaturated PAs.

revealed two unsaturated PAs (Fig. 8). The high concentration $(12000 \mu \mathrm{g} / \mathrm{g}$ ) of unsaturated PAs detected in the plant implicated this Senecio species as the cause of toxicity and the presence of the toxic PAs in the rumen content confirmed exposure of the cows. The diagnosis of PA poisoning was corroborated by histopathological evidence of diffuse centrilobular to submassive necrosis and haemorrhage in liver samples collected from the dead cows (Dimande et al., 2007).

The lack of commercially available reference materials is the major limitation in PA analysis. Retrorsine and monocrotaline were evaluated as reference standards as they are commercially available. The calibration curve of retrorsine was linear from 0.05 to $100 \mathrm{ug} / \mathrm{mL}$ and most of the compounds detected had retention times corresponding better with retrorsine than monocrotaline. The concentration of the unsaturated PAs in the samples were calculated against the retrorsine calibration curve and reported as $\mu \mathrm{g} / \mathrm{g}$ retrorsine equivalents. The method was developed to screen for unsaturated PA molecules, and standardization of results to retrorsine equivalents allowed for quantitative comparisons to be made between plants without first obtaining pure reference 
standards. This analytical method is an estimation of the relative concentration of toxic PAs present in a sample and not necessarily a reflection of the toxicity of the compounds.

\section{Conclusions}

A rapid screening method for the detection of unsaturated PAs in natural products was developed. The method specifically targets the 1,2 unsaturated necine bases responsible for different types of toxicity and can detect these compounds in very complex mixtures. The method reveals valuable information on the molecular masses of each of the unsaturated PAs as well as comparable concentrations which can be used to estimate the potential toxicity of the plant. The application of this method in evaluating the safety of plants ingested by animals, plant contaminants of food, herbal medicines and animal products such as honey and eggs could be useful.

\section{Conflict of interest}

The authors declare that there are no conflicts of interest.

\section{Acknowledgements}

The National Research Foundation provided funding and FARMOVS-Paraxel, Bloemfontein allowed us to use their instrumentation.

\section{References}

Australia New Zealand Food Authority. Pyrrolizidine alkaloids in food: a toxicological review and risk assessment. Technical report series no 2, Available from:<http://www.foodstandards.gov.au/_srcfiles/TR2.pdf>.

Dimande, A.F.P., Botha, C.J., Prozesky, L., Bekker, L., Rösemann, G.M., Labuschagne, L., Retief, E., 2007. The toxicity of Senecio inaequidens DC. J. S. Afr. Vet. Assoc. 78, 121-129. 
Eloff, J.N., Jansen van Rijssen, F.W., Naudé, T.W., Joubert, J.P.J., 2003. What levels of potentially toxic seed should be allowed in grains in South Africa? I. Background and pyrrolizidine alkaloid containing plants. S. Afr. J. Sci. 9, 337-334.

Fu, P.P., Xia, Q., Lin, G., Chou, M.W., 2002. Genotoxic pyrrolizidine alkaloids - mechanism leading to DNA adduct formation and tumorigenicity. Int. J. Mol. Sci. 3, 948-964.

International Programme on Chemical Safety. Pyrrolizidine alkaloids: health and safety guide. No 26. Available from:<http://www.inchem.org/documents/hsg/hsg/hsg026.htm>.

Kellerman, T.S., Coetzer, J.A.W., Naudé, T.W., Botha, C.J., 2005. Plant Poisonings and Mycotoxicoses of Livestock in Southern Africa, second ed. Oxford University Press, Cape Town.

Lin, G., Zhou, K.Y., Zhao, X.G., Wang, Z.T., But, P.P.H., 1998. Determination of hepatotoxic pyrrolizidine alkaloids by on-line high performance liquid chromatography mass spectrometry with electrospray interface. Rapid Commun. Mass Spectrom. 12, 1445-1456.

Mattocks, A.R., 1986. Chemistry and Toxicology of Pyrrolizidine Alkaloids. Academic Press, Orlando.

Ober, D., Hartmann, T., 1999. Homospermidine synthase, the first pathway-specific enzyme of pyrrolizidine alkaloid biosynthesis, evolved from deoxyhypusine synthase. P. Natl. Acad. Sci. USA. $96,14777-14782$.

Prakash, A.S., Pereira, T.N., Reilly, P.E.B., Seawright A.A., 1999. Pyrrolizidine alkaloids in human diet. Mutat. Res. 443, 53-67.

Wiedenfeld, H., 2011. Plants containing pyrrolizidine alkaloids: toxicity and problems. Food addit, and contamin. 28, 282-292. 